\section{Epidemiology and policy SP3-1 AN AUDIT OF DIABETES CARE AT PRIMARY HEALTHCARE
CENTERS IN DUBAI/UAE 2010}

doi:10.1136/jech.2011.142976o.1

B A Satar, ${ }^{*}$ H Hussain. Bushrasatar, Dubai, United Arab Emirates

Objectives To assess the current management of diabetic patients and benchmark the results against international standards.

Materials and Methods A retrospective medical record review of 87 registered diabetic patients was conducted in diabetes clinics, primary healthcare centers. Records were searched for the last 2 years of Audit implementation in Dubai concerning HbA1c, lipid, BMI, Systolic Blood pressure and diastolic blood pressure indicators in comparison with the standard auditing requirements as indicated by CDC, ICSI.

Results HBA1C was taken two or more times a year in $80.4 \%$ of the sample which meet the international standard recommendation and only one time in $16.6 \%$ of the sample per each year period, $67.82 \%$ of patients showed abnormal HBA1C results, $11 \%$ of the sample showed HBA1C $>9.5$ and $32.18 \%$ of patients showed HBA1C $<7$. BMI analysis showed that $89 \%$ of the patients were more than normal level (>25), 35\% were overweight while (25-30) and 54\% were obese according to BMI definition $(>30)$. The study showed that $46 \%$ of patients have systolic blood pressure $>130 \mathrm{~mm} \mathrm{Hg}$, and $29 \%$ showed diastolic blood pressure $>80 \mathrm{~mm} \mathrm{Hg}$. Lipid assessment was conducted on $93 \%$ of patients in the sample. The study showed significant difference $(p>0.05)$ from international standards in documentation for foot examination (22\%) of the sample, vision assessment (40\%) of the sample and documentation of advises on diet and exercise (35\%). As the international reference for good care and $(60 \%)$ for documentation.

Recommendation 1. Strengthening the healthcare delivery system in diabetes management to achieve the recommended standards as related to control the severity of the disease (which can be done through further investment in manpower, materials, logistics and whole management system. 2. Bringing down the level of.

\section{SP3-2 COMPARISON OF PESTICIDE EXPOSURE AND OCCUPATIONAL HEALTH ISSUES BETWEEN FULL TIME AND PART TIME VEGETABLE FARMERS IN THE PHILIPPINES}

doi:10.1136/jech.2011.142976o.2

J L Lu. ${ }^{*}$ National Institutes of Health, University of the Philippines Manila, Manila, The Philippines

Objectives This study aimed to compare the work practices and health effects of pesticide exposure between full time and part time vegetable farmers.

Methods Data gathering was done via structured personal interview using a nine page questionnaire, physical examination and blood extraction for complete blood count and serum creatinine.

Results Pyrethroid was the top pesticide type used by both groups. The risk for full time was related to both the amount of exposure and the type of pesticide the group is exposed to. There were more full time farmers who complained of getting or who fell ill because of work. This difference was statistically significant $(p=0.05)$. This health seeking behaviour was significantly different for both groups $(\mathrm{p}=0.01)$. In assessing for the individual component of the neurologic exam, $5.22 \%$ from full time farmers, and $8.63 \%$ from part time farmers had abnormal cranial nerve function, 22 (5.7\%) and $9(6.47 \%)$ have abnormal motor strength. All farmers tested for reflexes, meningeals, and autonomics from both groups were normal. For the haematologic examination, the full time farmers had higher mean values for creatinine, white blood cell, red blood cell, haemoglobin, and haematocrit. The activity of cholinesterase enzymes in the blood can be utilised as a biomarker for the effect of organophosphates. Out of the 232 blood cholinesterase results, 94 (40\%) were abnormal.

Conclusion The study showed certain differences between full time and part time farmers in terms of farming practices and health related problems. Education on safe pesticide use and handling and better health monitoring of the farmers are recommended.

\section{SP3-3 MULTIPLE INDICATOR CLUSTER SURVEY (MICS3): THE SITUATION AND DISPARITIES OBSERVED IN ALGERIA}

doi:10.1136/jech.2011.1429760.3

0 Boualem, ${ }^{*} \mathrm{~K}$ Houria. Department of population and health, Boumerdes, Algeria

Introduction This presentation contains the main results of the third Multiple Indicator Cluster Survey (MICS3) of Algeria, which was conducted in 2006 by the National Office of Statistics and the Ministry of Public Health. This investigation is an international initiative launched by Unicef to support countries to fill gaps in information on human development and the situation of children and women. This is the third survey conducted in Algeria (previously undertaken in 1995 and 2000).

Objectives 1 . To provide the data required to monitor the Millennium Development Goals. 2. To provide data to enable public authorities to implement, monitor and evaluate health policies. 3 . To provide regional indicators and to analyse the regional disparities and implement programs to reduce them. 4. To provide data for comparison with other countries.

Methods and Materials A sample size calculation was undertaken in order to allow results to be representative at national and regional levels, and also according to urban and rural strata. A total of 29478 households spread across 17 sub-regions were selected, consisting of 47612 women (age 15-49 years) and 15000 children (age $<5$ years). Results Core data collected for children includes: nutrition, breastfeeding, vaccination, diarrhoeal diseases, education, discipline of children, and birth registration. Data for women includes: general characteristics, marital status, fertility, family planning, maternal care, and knowledge about the transmission of HIV/AIDS. Data for the general population includes: chronic diseases, physical and mental handicap.

Conclusion The observed results demonstrate the improvement of the general population in a number of domains, however, differences persist. These results have pushed the government to adapt the programs, taking account of regional and local differences.

\section{SP3-4 INCIDENCE OF NASOPHARYNGEAL CARCINOMA IN THE NORTH OF TUNISIA: TENDENCY AND PROJECTIONS UNTIL 2024}

doi:10.1136/jech.2011.1429760.4

${ }^{1}$ W H B Ayoub, ${ }^{2} \mathrm{H}$ Rais, ${ }^{*}{ }^{1} \mathrm{~S}$ Zehani, ${ }^{3} \mathrm{H}$ Hamadi, ${ }^{1} \mathrm{M}$ B Abdallah. ${ }^{1}$ Department of Epidemiology and bio statistics, Salah Azaiez Institute of oncology, Tunis, Tunisia; ${ }^{2}$ Medical Oncology Department, Salah Azaiez Institute of oncology, Tunis, Tunisia; ${ }^{3}$ National Institute of Public Health, Tunis, Tunisia

Nasopharyngeal carcinoma (NPC) is the second neoplasm of ORL site in Tunisia with a bimodal distribution and a first period occurrence between 15 and 20 years old and a second period occurrence at 50 years old. Data of cancer registry of North Tunisia (CRNT) of the period 1999-2003 confirmed that Tunisia is an intermediate risk area for NPC. This study aims to present the evolution of incidence rate of NPC over a period of 10 years (1994-2003) and try to establish a projection of this rate and the number of cases on 2024 in the north of Tunisia. 